\title{
Container assignment optimization considering overlapping amount and operation distance in rail-road transshipment terminal
}

\author{
Wang, L. ${ }^{a,}{ }^{,}$, Zhu, X. ${ }^{a}$, Xie, Z. ${ }^{a, b}$ \\ ${ }^{a}$ School of Traffic and Transportation, Beijing Jiaotong University, Beijing, P.R. China \\ ${ }^{\mathrm{b}}$ State Key Laboratory of Rail Traffic Control and Safety, Beijing Jiaotong University, Beijing, P.R. China
}

\section{A B S T R A C T}

Container assignment strategy is crucial to the operation efficiency of railroad container transshipping system. An effective container assignment approach can markedly improve integral operation efficiency of rail-road container transshipping system. In this paper, the container assignment problem in rail-road transshipment terminal was described and formulated as a twostage optimization model considering overlapping amount and operation distance of crane. The first stage optimization model was to optimize container assigning positions for minimizing the total overlapping amount caused by container assigned in the considered block at one planning period, and an iterative solution procedure was proposed to obtain container assignment sets. Based on the container assignment sets obtained by the first stage, the second stage optimization model was to optimize the container assigning sequence for decreasing the total operation distance of crane, and a genetic algorithm was designed to obtain the optimal container handling sequences in container assignment process. Computational experiments on the data from a rail-road transshipment terminal in China were implemented to test efficiency of the proposed approach. Computational results showed that the proposed approach was effective to reduce overlapping amount and operation distance in container assignment process. The proposed approach is significant for the production and management of rail-road container transshipping terminals.
\end{abstract}

\section{ARTICLE INFO}

Keywords:

Intermodal transportation

Container assignment

Terminal scheduling

Rail-road transshipment terminal

Optimization

Genetic algorithms

*Corresponding author:

liwang@bjtu.edu.cn

(Wang, L.)

Article history:

Received 25 January 2017

Revised 21 October 2017

Accepted 26 October 2017

(C) 2017 PEI, University of Maribor. All rights reserved.

\section{References}

[1] Boysen, N., Fliedner, M. (2010). Determining crane areas in intermodal transshipment yards: The yard partition problem, European Journal of Operational Research, Vol. 204, No. 2, 336-342, doi: 10.1016/i.ejor.2009.10.031.

[2] Bazzazi, M., Safaei, N., Javadian, N. (2009). A genetic algorithm to solve the storage space allocation problem in a container terminal, Computers \& Industrial Engineering, Vol. 56, No. 1, 44-52, doi: 10.1016/i.cie.2008.03.012.

[3] Kim, K.H., Park, K.T. (2003). A note on a dynamic space-allocation method for outbound containers, European Journal of Operational Research, Vol. 148, No. 1, 92-101, doi: 10.1016/S0377-2217(02)00333-8.

[4] Zhang, C., Wu, T., Zhong, M., Zheng, L., Miao, L. (2014). Location assignment for outbound containers with adjusted weight proportion, Computers \& Operations Research, Vol. 52, 84-93, doi: 10.1016/i.cor.2014.06.012.

[5] Tang, L., Sun, D., Liu, J. (2016). Integrated storage space allocation and ship scheduling problem in bulk cargo terminals, IIE Transactions, Vol. 48, No. 5, 428-439, doi: 10.1080/0740817X.2015.1063791.

[6] Yu, M., Qi, X. (2013). Storage space allocation models for inbound containers in an automatic container terminal, European Journal of Operational Research, Vol. 226, No. 1, 32-45, doi: 10.1016/i.ejor.2012.10.045.

[7] Sharif, O., Huynh, N. (2013). Storage space allocation at marine container terminals using ant-based control, Expert Systems with Applications, Vol. 40, No. 6, 2323-2330, doi: 10.1016/i.eswa.2012.10.032. 
[8] Hu, W., Wang, H., Min, Z. (2014), A storage allocation algorithm for outbound containers based on the outerinner cellular automaton, Information Sciences, Vol. 281, 147-171, doi: 10.1016/i.ins.2014.05.022.

[9] Boysen, N., Fliedner, M., Jaehn, F., Pesch, E. (2013). A survey on container processing in railway yards, Transportation Science, Vol. 47, No. 3, 312-329, doi: 10.1287/trsc.1120.0415.

[10] Wang, L., Zhu, X., Xie, Z. (2014). Storage space allocation of inbound container in railway container terminal, Mathematical Problems in Engineering, Vol. 2014, 1-10, doi: 10.1155/2014/956536.

[11] Wang, L., Zhu, X.-N., Yan, W., Xie, Z.-Y., Li, Q.-B. (2013). Optimization Model of Mixed Storage in Railway Container Terminal Yard, Journal of Transportation Systems Engineering and Information Technology, Vol. 2, 172-178, doi: 10.3969/j.issn.1009-6744.2013.02.026.

[12] Guo, P., Cheng, W., Zhang, Z., Zhang, M., Liang, J. (2013). Gantry crane scheduling with interference constraints in railway container terminals, International Journal of Computational Intelligence Systems, Vol. 6, No. 2, 244-260, doi: $10.1080 / 18756891.2013 .768444$.

[13] Wang, L., Zhu, X., Xie, Z. (2016). Rail mounted gantry crane scheduling in rail-truck transshipment terminal, Intelligent Automation \& Soft Computing Vol. 22, No. 1, 61-73, doi: 10.1080/10798587.2015.1041764.

[14] Tang, M., Gong, D., Liu, S., Zhang, H. (2016). Applying multi-phase particle swarm optimization to solve bulk cargo port scheduling problem, Advances in Production Engineering \& Management, Vol. 11, No. 4, 299-310, doi: 10.14743/apem2016.4.228.

[15] Grubišić, N., Dundović, Č., Žuškin, S. (2016). A split task solution for quay crane scheduling problem in mid-size container terminals, Tehnički vjesnik - Technical Gazette, Vol. 23, No. 6, 1723-1730, doi: 10.17559/TV20150914110215.

[16] Rajkovic, R., Zrnic, N. Stakic, D. (2016). Application of a mathematical model for container transport flow of goods: From the Far east to Serbia, Tehnički vjesnik - Technical Gazette, Vol. 23, No. 6, 1739-1746, doi: 10.17559/ TV-20140629203730. 


\title{
Optimizacija razporejanja kontejnerjev v železniško-cestnem tovornem terminalu $z$ upoštevanjem prekrivanja in obratovalne razdalje
}

\author{
Wang, L., ${ }^{a}{ }^{*}$, Zhu, X. ${ }^{a}$, Xie, Z. ${ }^{a, b}$ \\ ${ }^{a}$ School of Traffic and Transportation, Beijing Jiaotong University, Beijing, P.R. China \\ ${ }^{\mathrm{b}}$ State Key Laboratory of Rail Traffic Control and Safety, Beijing Jiaotong University, Beijing, P.R. China
}

\section{POVZETEK}

Strategija razporejanja kontejnerjev je ključnega pomena za učinkovitost obratovanja pretovarjanja kontejnerjev v pristaniščnih železniško-cestnih terminalih. Učinkovit pristop pri razporejanju kontejnerjev lahko bistveno izboljša učinkovitost delovanja takšnega sistema. V tem prispevku je opisan problem pri razporejanju kontejnerjev v terminalu za pretovarjanje. Problem je preoblikovan $v$ dvostopenjski optimizacijski model, ki upošteva prekrivanje in obratovalno razdaljo žerjava. V prvem koraku se izvede optimizacija razporejanja kontejnerjev z namenom minimiziranja prekrivanja, ki ga povzroči kontejner, razporejen na določeno mesto za določeno časovno obdobje. Za razporeditev skupine kontejnerjev je uporabljen iterativni postopek. Na podlagi razporeditve skupin kontejnerjev iz prvega koraka se v drugem koraku z genetskim algoritmom optimizira zaporedje razporejanja kontejnerjev za zmanjšanje skupne obratovalne razdalje žerjava. Za računske preizkuse so bili uporabljeni realni podatki iz kitajskih tovornih terminalov, rezultati pa so potrdili učinkovitost algoritma pri zmanjšanju stopnje prekrivanja in obratovalne razdalje žerjava v procesu razporejanja kontejnerjev. Predlagan pristop je pomemben za proizvodnjo in vodenje železniško-cestnih pristaniščnih terminalov.

\section{PODATKI O ČLANKU}

Ključne besede:

Medfazni transport

Dodeljevanje kontejnerjev

Načrtovanje obratovanja terminala

Železniško-cestni tovorni terminal

Optimizacija

Genetski algoritmi

* Kontaktna oseba: liwang@bjtu.edu.cn (Wang, L.)

Zgodovina članka:

Prejet 25. januarja 2017

Popravljen 21. oktobra 2017

Sprejet 26. oktobra 2017 